ISSN 1409-2441

www.ucr.ac.cr
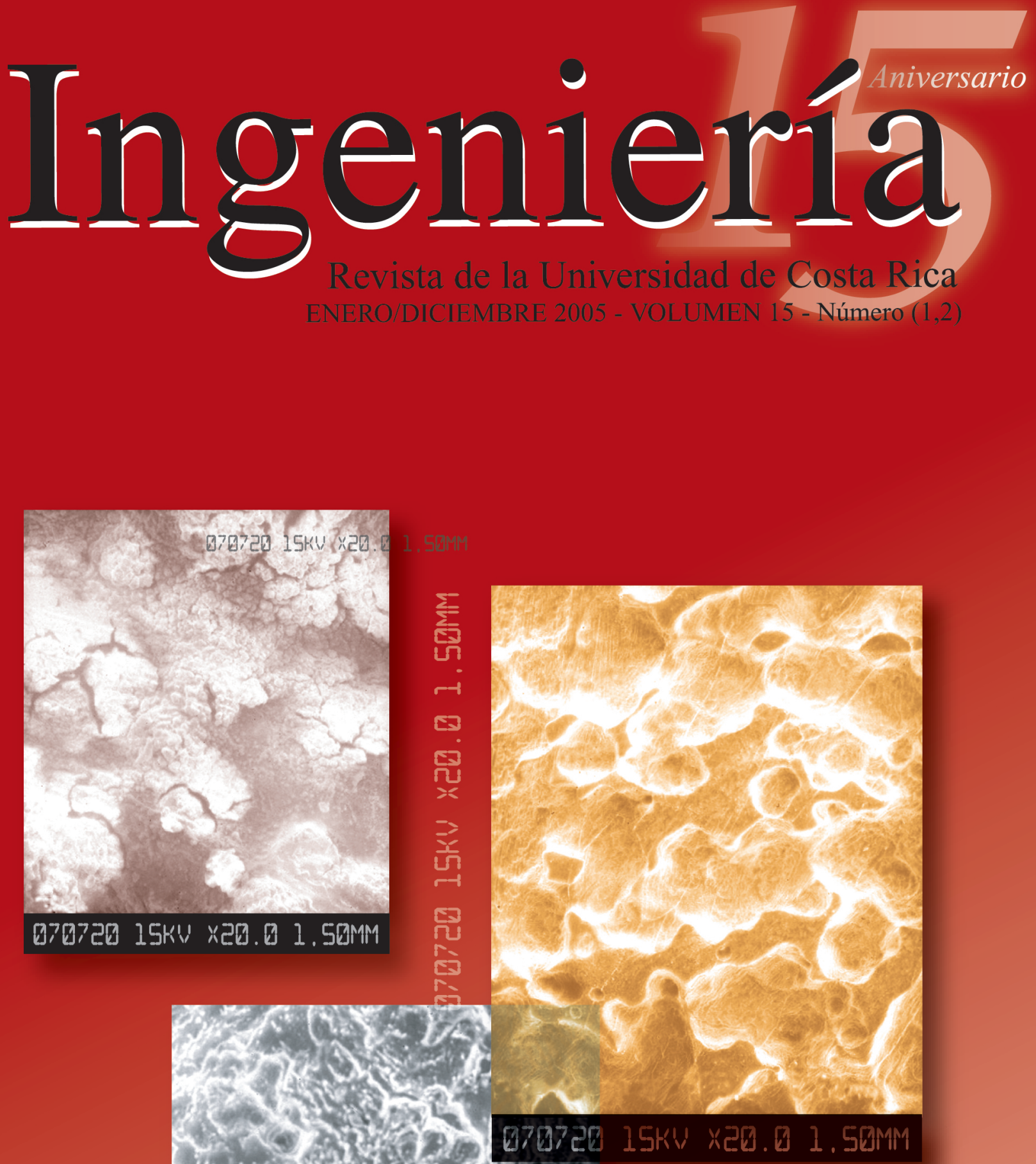

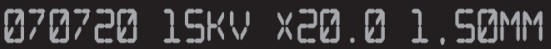

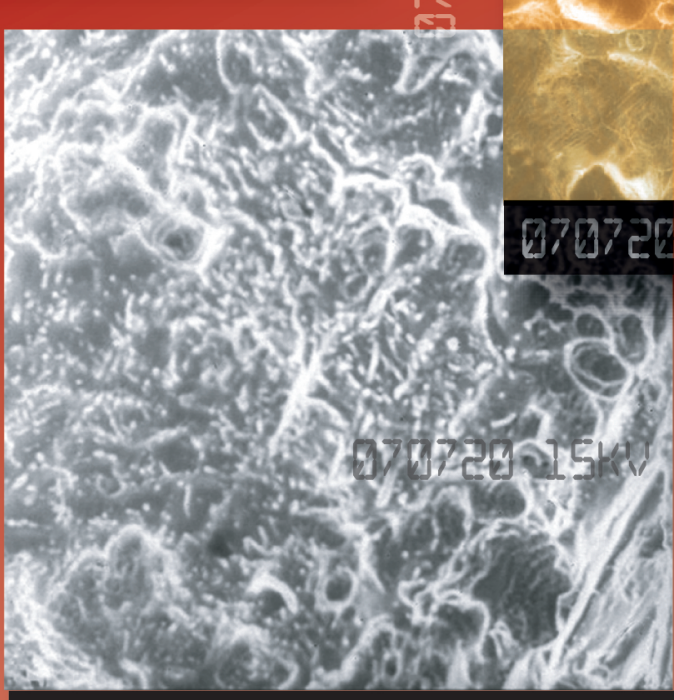

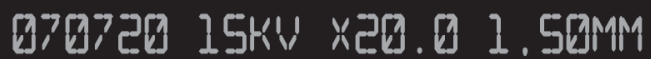




\title{
DESARROLLO SOSTENIBLE: UNA PLATAFORMA NECESARIA EN EL CURRÍCULO DE LAS CARRERAS DE INGENIERÍA

\author{
Herminia Casanueva López.
}

\begin{abstract}
Resumen
Se presenta un análisis de diveros indicadores que revelan el deterioro de algunas variables medioambientales del planeta y de Costa Rica; a partir de este diagnóstico, se analizan algunas alternativas para alcanzar un modelo de desarrollo sostenible a través de la educación, tales como la Declaratoria del Decenio para el Desarrollo Sostenible, declarado por Naciones Unidas y la Declaración de Taillores suscrita por la Asociación de Universidades Líderes para un Futuro Sostenible.
\end{abstract}

Con estos antecedentes, se proponen algunas recomendaciones para la reformulación de los currículos de las instituciones de educación superior, específicamente de las carreras de ingeniería.

Palabras clave: educación, sostenibilidad, ingeniería, currículo.

\section{Abstract}

An analysis of some indicators reveal the deterioration of some environmental variables of the planet and of Costa Rica, from this diagnose, some alternatives are analyzed to reach a model of sustainable development though the education, such as the declaration of the Decade for the Sustainable Development declared by United Nations and the Declaration of Taillores subscribed by the Association of Universities Leaders for a Sustainable Future.

With these antecedents some recommendations for the reformulation of the curricula of the institutions of superior education and of engineering specifically.

Key words: education, sustainability, engineering, curriculum.

Recibido: 08 de julio del 2005 / Aprobado: 30 de agosto del 2005

\section{UNA VISIÓN DEL CONTEXTO}

Antes de plantear una propuesta para revisar el currículo de una carrera universitaria, la primera tarea debe ser ubicar el contexto en el cual ella se desarrolla, empezando por lo más general hasta llegar a las consideraciones particulares de su entorno inmediato.

Cuando el capitán de la nave Apollo XIII informó a la estación espacial de Houston que habían estallado los tanques de oxígeno, inició su informe con una frase que se haría famosa: "Houston, tenemos un problema", con estas breves palabras resumió la gravedad de la situación que describiría a continuación. Haciendo una paráfrasis de esta expresión, hoy, treinta y cinco años después, viendo el estado del planeta y de quienes en él vivimos, podemos decir: "Houston, tenemos problemas", pero la diferencia es que ahora los problemas los tenemos nosotros, la Tierra y sus habitantes.

Revisemos datos que reflejan el deterioro del estado del planeta y la evolución de algunos indicadores en los últimos años: 


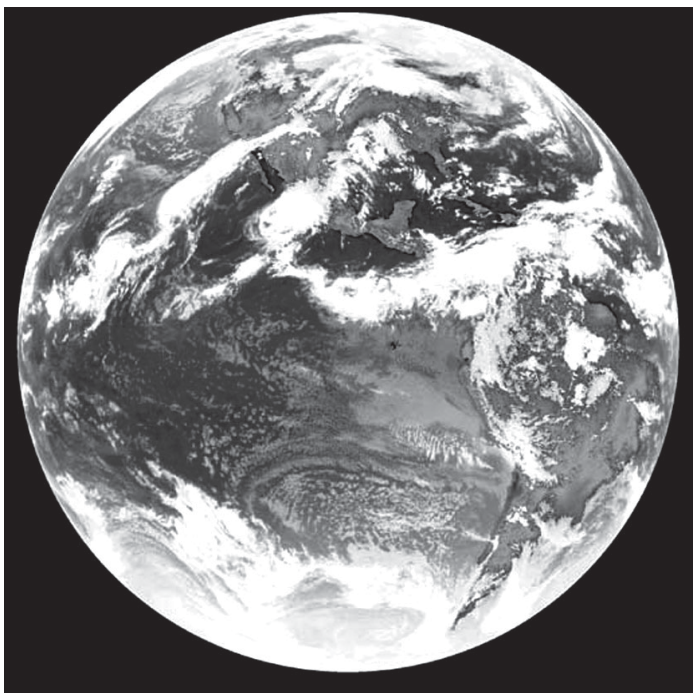

Fuente: (Observatorio de la Nasa)

"Houston: Tenemos un problema" Capitán Al Sheppard

Apollo XIII, 13 de abril de 1970.

- Desde 1950 a la fecha se ha perdido el $50 \%$ de los pantanos y de los bosques del planeta (UNPD, 2000: p. 14).

- $\quad$ El $70 \%$ de la vida marina está en peligro (Ibíd.: p. 12).

- El $65 \%$ de las tierras agrícolas presenta grave degradación de los suelos (Ibíd., p: 8).

- El agua potable disponible por persona se ha reducido en un 50 \% (Ibíd., 1998: p. 4).

- $\quad$ El $20 \%$ de la población mundial carece de acceso al agua (Ibíd., p. 2).

- Se calcula que la temperatura promedio de la superficie terrestre se incrementará entre $1,4^{\circ} \mathrm{C}$ y $3,5^{\circ} \mathrm{C}$ en los próximos 100 años (IPCC WGI, 2001: p. 8).

- La concentración de $\mathrm{CO}_{2}$ en la atmósfera se ha incrementado en un $31 \%$ desde 1750; la tasa actual de incremento no tiene precedentes en los últimos 20000 años (Ibíd., p. 4).
- Producir $100 \mathrm{~kg}$ de productos industriales genera $3200 \mathrm{~kg}$ de basura (Ryan, 1997: p. 4).

- Se encuentran en peligro inmediato de desaparición (UNPD, 1998; p. 48): el $11 \%$ de las 8615 especies de pájaros; el $12 \%$ de las 4355 especies de mamíferos y el $29 \%$ de todas las especies de peces.

- Se presenta un aumento de los problemas de salud, contaminación de las fuentes de agua, incremento en los niveles de contaminación del aire, así como un incremento de las enfermedades producidas por vectores asociados al cambio climático (USEPA, 2000: p. 3).

- La brecha entre el segmento más rico de la humanidad y el más pobre se ha incrementado. La tasa de ingreso del $20 \%$ más rico, en relación con el $20 \%$ más pobre de la humanidad, ha pasado de una relación de 30:1 en 1960, a un 92:1 en 1995 (UNPD, 1998: p. 29).

- Somos la primera generación con la capacidad de determinar la habitabilidad del planeta para los seres humanos y para las otras especies que lo habitan, sean animales o vegetales (Cortese, 1970, p: 3).

Dentro de este panorama global, cada país presenta su propia problemática que se suma a la planetaria y crea condiciones particulares que deben resolverse local, regional y mundialmente. Algunos datos permiten tener una idea de la situación de deterioro ambiental en Costa Rica.

- El volumen de basura generado se ha duplicado en los últimos veinte años, y la mayor parte de ella permanece sin recibir ningún tipo de tratamiento (Estado de la Nación, 2001: p. 64).

- De las aproximadamente 3500 industrias establecidas en la cuenca del golfo de Nicoya, solo el $5 \%$ tiene planta de tratamiento de aguas (Ibíd., p. 64). 
- El $33 \%$ de la tala ilegal se da en fincas agrícolas o ganaderas, sin embargo, por tratarse de una deforestación críptica, esta cifra podría ser mayor (Ibíd., p. 65).

- $\quad$ En el 2001 se informó sobre dos episodios de intoxicación masiva en la Gran Área Metropolitana, debidos a la contaminación de fuentes superficiales de agua. La ausencia de medidas de protección de fuentes y zonas de captación estratégica y de control y reducción de la contaminación, se han transformado en riesgos silenciosos de escasez de agua de calidad, degradación ambiental y problemas de salud pública (Ibíd., p. 65).

- El consumo de agua en la Gran Área Metropolitana se duplicó en cuatro años. Allí existen cerca de 5000 pozos registrados y un número indeterminado de pozos ilegales, se desconoce el estado de los acuíferos (Ibíd., p. 66).

- $\quad$ El $72 \%$ de las inundaciones y el $74 \%$ de los deslizamientos registrados en el año 2001 no tuvieron relación con eventos hidrometeorológicos extremos. El $23 \%$ de las inundaciones se relacionó con problemas de alcantarillado urbano o inadecuados movimientos de tierras, acumulación de basura o urbanización en áreas no aptas (Ibíd., p. 66).

- Se calcula que de las 12000 especies de plantas que se encuentran en el territorio nacional, entre un $10 \%$ a un $12 \%$ se encuentran amenazadas y del total de la flora, poco más del $25 \%$ se considera rara debido a que es escasa o poco conocida a causa de la deforestación (INBIO, 2005).

- En la última década, el consumo de hidrocarburos se ha incrementado en $45,5 \%$, principalmente debido al aumento de la flota vehicular y a la subutilización del transporte de carga (Chacón, 2002, p: 1).

- El consumo nacional de energía según su origen en el año 1990 fue: un $15 \%$ en electricidad, un $46 \%$ en derivados de petróleo y un $39 \%$ en biomasa, mientras que para el año 1999, el consumo nacional se distribuyó de la siguiente manera: $20 \%$ electricidad, $72 \%$ derivados de petróleo y 8 $\%$ biomasa (Chin-Wo, 2001).

\section{EL SECTOR PRODUCTIVO}

Una de las características básicas del modelo económico imperante en el mundo es la que se basa en la premisa de que se requiere un crecimiento constante para sostenerse; lógicamente, para mantener una tasa de crecimiento constante se requieren cada vez más recursos, lo que hace necesaria una explotación también creciente de los recursos naturales, si se considera que estos son, por su misma naturaleza, finitos y no renovables en el corto plazo, resulta entonces obvia la falta de sostenibilidad de este modelo (Capra, 1985: p. 243).

A pesar de la abundante evidencia que muestra que los altos índices de consumo no resuelven ninguno de los problemas ambientales y sociales más urgentes y que más bien se acompañan de un deterioro de la calidad de vida de sectores crecientes de la población, se gastan enormes sumas de dinero en publicidad para mantener altos los niveles de consumo de bienes y servicios que en muchos casos son innecesarios, excesivos y con frecuencia altamente perjudiciales (Ibíd., p. 245).

Mientras las multinacionales intensifican su búsqueda de recursos naturales, de mano de obra barata y de nuevos mercados en todo el mundo, los desastres ecológicos y las tensiones sociales creadas en su afán de crecimiento infinito, se hacen cada vez más evidentes.

Una noción básica en economía es el principio de que tanto el capital como la mano de obra engendran riqueza, pero una economía basada en el capital también supone un uso intensivo de recursos naturales y de energía, y por ello resulta finalmente inflacionaria e insostenible.

Uno de los campos en que es más evidente y clara esta relación entre economía y recursos naturales es la agricultura y la producción de 
alimentos en general. La agricultura moderna, la que se enseña en nuestras universidades latinoamericanas, tiene como referente principal el modelo norteamericano, que se encuentra basado en un uso intensivo de la tierra con el apoyo de maquinaria operada con combustibles fósiles y sistemas de irrigación intensivos en el uso de energía, y que además también depende de fuertes dosis de agroquímicos elaborados a base de petróleo ( Ibíd., p. 237).

Estos métodos y técnicas de explotación destruyen el equilibrio natural de los sistemas y producen sustancias tóxicas que se incorporan al suelo y a los alimentos, envenenando nuestros cuerpos y generando un agotamiento creciente de los suelos, que cada vez dependen más de los agroquímicos para mantener los niveles de producción, con lo cual hacen a los agricultores responsables y víctimas del proceso inflacionario.

La industria alimentaria está basada en el aumento del valor agregado de los productos mediante sucesivos procesos y transformaciones tecnológicas que incluyen operaciones como limpieza, selección, empaque, refrigeración, transporte, enlatado, congelado, diversos procesos de preparación y precocción. Todos estos procesos requieren cantidades crecientes de energía y además, para transportar los alimentos manteniéndolos en las mejores condiciones de higiene y frescura, se requieren cantidades crecientes de material de empaque, el cual una vez que el producto a su punto de destino, se convierte inmediatamente en basura y por lo tanto, también estimulan la inflación y contribuyen a agotar los recursos.

Algunos de los sistemas agrícolas más tecnificados valga decir mecanizados son de los más ineficientes del mundo, si se les juzga desde el punto de vista de la cantidad de energía utilizada para producir un determinado número de calorías; sin embargo, las empresas agrícolas de los países denominados desarrollados, obtienen grandes ganancias a través de los subsidios y otros mecanismos como cuotas o mecanismos de protección de mercados. Este sistema que utiliza una enorme cantidad de los recursos no renovables del planeta para el beneficio de unos pocos, debe considerarse extremadamente ineficaz, ecológica y económicamente (Capra, Ibid.: p. 263).

Con la introducción de los agroquímicos durante la llamada "Revolución Verde" de los años sesentas, la agricultura se mecanizó y se hizo más dependiente de la energía, lo que favoreció a los terratenientes con capital para invertir y obligó a los pequeños propietarios a vender sus tierras, abandonar el cultivo de las variedades locales y migrar a las ciudades o volverse peones asalariados en el lugar donde fueron sus tierras.

Junto con la mecanización y el monocultivo también se introdujo el uso masivo de agroquímicos, sin embargo hasta ahora, la agricultura química no ha ayudado ni a los agricultores, ni a la tierra, ni a los consumidores Se calcula que una persona que viva actualmente, posee en su organismo más de 500 sustancias químicas sintéticas que no existían antes de 1920 (Halweil: 2002; p. 45).

Lamentablemente, no parece que la industria agroquímica haya aprendido las lecciones de la Revolución Verde. Ehrenfeld, 1997: p. 36, indica que: "Al igual que ocurre con la agricultura intensiva, se trata a menudo de presentar a la ingeniería genética como una tecnología humana, dedicada a alimentar a la gente más y mejor. Nada más lejos de la realidad. Con escasísimas excepciones, el único objetivo de la ingeniería genética consiste en aumentar las ventas de productos químicos y transgénicos a unos agricultores cada vez más dependientes."

Por otro lado, Capra en su obra, "Las Conexiones Ocultas", (Capra, 2003: p. 240) resume claramente el panorama actual de la producción de alimentos: "A través de una serie de fusiones masivas, y gracias al estrecho control permitido por las tecnologías genéticas, se está produciendo una concentración de propiedad y control sobre la producción de alimentos sin precedentes. Las diez empresas agroquímicas más grandes controlan ya el ochenta y cinco por ciento del mercado global, mientras que las cinco primeras controlan prácticamente la totalidad del mercado de las semillas." 
Uno de los argumentos que más frecuentemente esgrimen las transnacionales sobre los alimentos, es que la ingeniería genética resolverá el problema del hambre en el mundo; en 1998, la publicidad de Monsanto en los medios de comunicación afirmaba que: "Preocuparse por el hambre de las generaciones futuras no les va a dar de comer. La biotecnología alimentaria sí”. Nada más lejos de la verdad; para muestra un botón: la gran mayoría de los niños mal nutridos del mundo, menores de cinco años, viven en países que exportan alimentos (UNICEF, 1998).

El problema del hambre no es técnico, es político; las causas del hambre no tienen que ver con la producción de alimentos, sino con la pobreza, con la desigualdad de oportunidades, con la injusta distribución de la tierra con vocación agrícola y con prácticas agrícolas que buscan optimizar las ganancias a costas del ambiente y no de la producción de alimentos.

Una de las exigencias más fuertemente sostenida por los organismos financieros internacionales sugiere que los países pobres deben buscar nichos de mercado, oportunidades competitivas y centrar sus esfuerzos en unos pocos bienes específicos para la exportación, a fin de obtener divisas y así comprar el resto de los bienes y servicios que requieran. Este planteamiento genera una gran dependencia, desequilibrio y vulnerabilidad, pues la economía de todo un país depende de muy pocos productos, esta situación se agrava en aquellos países que no poseen recursos naturales exportables como petróleo o minerales, tal es el caso de Costa Rica, que paga una parte importante de su factura petrolera con exportaciones agrícolas.

Otro problema de este modelo económico es la gran presión que ejerce sobre los recursos naturales, acelerando su agotamiento; la ampliación de las áreas dedicadas a ciertos cultivos, que como la caña de azúcar y la piña, requieren grandes cantidades de agua y han puesto en riesgo los acuíferos que alimentan los sistemas de riego, pues extraen el agua a una velocidad mayor que la capacidad de recarga.
Adicionalmente, las prácticas de monocultivo extensivas también contaminan los mantos subterráneos por el arrastre de agroquímicos usados en las plantaciones, hacia capas más profundas del suelo, muchas veces a una gran distancia del punto de contaminación; la constatación de esta situación ha llevado a afirmar que: "Buenos suelos agrícolas se han agotado al ser dedicados a cultivos de plantación dedicados a conseguir dólares” (Capra, 2003: p. 192).

\section{3. ¿ES POSIBLE UN MUNDO SOSTENIBLE?}

Ante este panorama aparentemente desalentador, es necesario preguntarse qué se puede hacer, quiénes deben hacerlo y, si aún es posible, hacer algo para asegurar la sostenibilidad del planeta.

Para iniciar la construcción de un nuevo paradigma, es necesario impulsar una reforma educativa sostenible y con visión de futuro; el modelo actualmente vigente se sustenta en la reproducción de la injusticia y el abuso de los recursos naturales, es necesario sustituir este esquema e introducir uno basado en nuevos principios; en este sentido, la Organización de las Naciones Unidas (ONU) en su Asamblea celebrada el 22 de diciembre de 2002, aprobó bajo el número 57/254, una resolución para establecer la "Década para la Educación para el Desarrollo Sostenible" que va del $1^{\circ}$ de enero del 2005 al 31 de diciembre del 2014 (Anexo A).

Esta Declaratoria señala claramente que la educación es un elemento indispensable para alcanzar el desarrollo sostenible e impulsa aquellas iniciativas, en todo el mundo, que incluyan el estudio y la solución de problemas locales, mediante la aplicación de los principios del Desarrollo Sostenible en diferentes situaciones de enseñanza y aprendizaje. Señala también que el avance hacia el desarrollo sostenible depende de las acciones de los ciudadanos en todos los aspectos de la vida, lo que supone adoptar cambios en la manera de pensar, vivir y relacionarse con el ambiente. Partiendo de esta consideración, 
se establecen marcos de actuación que facilitan la participación, la toma de conciencia y la educación.

Para conseguir el desarrollo sostenible es necesario que cada vez haya más gente preocupada por las consecuencias sociales y ambientales de sus actuaciones y que, además, cuenten con la formación y la motivación para cambiar sus hábitos de vida y de trabajo en procura de los objetivos de la sostenibilidad.

Los aspectos más importantes que cubre la declaración de la ONU sobre Educación para la Sostenibilidad son: (CENEAM, 2005)

- Conocimiento por parte del público de los principios en los que se asienta la sostenibilidad, con una visión clara, donde lo relevante es cómo se aplica en la práctica a los distintos contextos culturales y locales.

- Participación de todos los sectores sociales, incluidas empresas, sector agrícola, turismo, gestores de recursos naturales, gobiernos locales, medios de comunicación, sector educativo y otras fuerzas de la sociedad civil.

- Fomentar una educación continua y de calidad para todos los individuos, independientemente de sus obligaciones y circunstancias. Sin el acceso a una educación básica e incluso superior, los pueblos tendrán pocas posibilidades de alcanzar un desarrollo que sea sostenible.

- Reorientar los programas educativos existentes en todos los países hacia los conocimientos económicos, sociales y ambientales, las destrezas y valores inherentes a la sostenibilidad.

- Crear programas de formación específicos dirigidos a los sectores más afectados que los capaciten para alcanzar una mejor calidad de vida y un desarrollo sostenible.

La Declaratoria aborda la problemática de la sostenibilidad desde una perspectiva inclusiva y de largo plazo, por lo que se define claramente que la educación es el mecanismo para lograrlo. Por esta razón se designó a UNESCO (Organización de las Naciones Unidas para la Educación, la Ciencia y la Cultura), como la responsable de proponer y coordinar el plan de acción para el buen desarrollo de la década.

Entre los temas que se han propuesto se incluyen: reducción de la pobreza, igualdad de género, tecnologías para la sostenibilidad, medio ambiente, agua, derechos humanos, diversidad cultural, reducción de desastres y urbanización sostenible; con esta enumeración se puede apreciar que la Declaratoria propone que se debe asumir un compromiso para que toda la educación preste atención a los problemas del planeta y de quienes en él habitamos, desde una perspectiva sistémica que incluya todos los aspectos relativos a la vida en la Tierra (OEI, 2005).

\section{FUNDAMENTOS DEL NUEVO CURRÍCULO}

Una educación comprometida con la sostenibilidad, debe trabajar para que los habitantes del planeta tengan sus necesidades básicas satisfechas, un acceso equitativo a los recursos naturales, una calidad de vida que permita el disfrute del arte la recreación y la cultura, y que con su actuar, preserven la diversidad cultural, biológica y los ecosistemas de los que dependen todas las formas de vida. El paradigma de la sostenibilidad incluye ricas y atractivas alternativas para el cambio de nuestros patrones de conducta, pero un cambio hacia la sostenibilidad supone algo más que una visión de "solución de problemas", implica un cambio hacia una visión de "creación" de un nuevo futuro. Una visión basada en la identificación de problemas implica que eliminar los problemas conlleva lograr las metas que se desean, pero esto no es necesariamente cierto. Por otra parte, el concepto de creación supone traer algo que se desea y darle existencia (Cortese, 1999: p. 3).

Crear un futuro sostenible supone qué somos capaces de definir en términos concretos que 
quiere decir sostenibilidad en todos los ámbitos de nuestra vida. Nuestra manera de pensar y actuar debe ser revisada para enfrentar los valores impulsados por una economía globalizada basada en el consumo, que es uno de los principales obstáculos para lograr la sostenibilidad; citando a Einstein, (2005), se puede afirmar que: los problemas significativos que enfrentamos no pueden ser resueltos en el mismo nivel de pensamiento que teníamos cuando los creamos.

Al sistema educativo actual le cabe parte de la responsabilidad, pues hasta ahora no se ha comprometido verdaderamente con la problemática ambiental, cuando incursiona en ella lo hace de manera tangencial y puntual, con una visión de especialista, sin que se le presente como un contenido transversal que afecta la concepción misma del currículo, porque atañe a todas las esferas de la vida en el planeta, esto es especialmente grave en las instituciones de educación superior. En este sentido, como señala (Cortese, 1999: p 6), al igual que los centros más altos de enseñanza, investigación y aprendizaje, las instituciones de educación superior tienen suficiente información y conocimientos para contribuir al cambio de la sociedad hacia un modelo más sostenible de desarrollo, sin embargo, hasta ahora los más prestigiosos centros de conocimiento han contribuido a incrementar el colapso ecológico.

Capitalizar la influencia que las universidades poseen en la sociedad en beneficio de un futuro sustentable requiere cambios significativos en la labor académica y dentro de la gestión de las instituciones, cuanto más aprendemos cómo la actividad humana está degradando el ambiente y generando cambios en los sistemas climáticos, menos se justifican las "soluciones" puntuales que no involucran un cambio conceptual radical en las bases del sistema educativo y especialmente entre quienes tienen la responsabilidad de formar líderes para el futuro.

La educación superior ha sido muy lenta en responder a las demandas de la sostenibilidad, debido, entre otros factores, al modelo de compartimentación de saberes que la caracteriza.
Un problema fundamental ha sido la tendencia a tratar la educación para la sostenibilidad como un problema puntual, cuya solución es la formación de especialistas en distintos ámbitos, la formación de especialistas no es la respuesta adecuada a la problemática que enfrentamos.

Una educación dividida en compartimentos refuerza la suposición de que la protección del ambiente y la sostenibilidad en general, son un asunto de especialistas, obviando que todos los seres humanos consumimos recursos, producimos basura, e interactuamos con el ecosistema en todas las actividades de nuestra vida; es nuestra manera de interactuar entre nosotros y con el ambiente, lo que debe ser modificado.

Las instituciones de educación superior tienen especial responsabilidad en incrementar las fortalezas de la sociedad para crear un futuro justo y sustentable para todos. En este sentido, ya se han iniciado algunos esfuerzos entre los que conviene destacar, a modo de ejemplo, la "Declaración Talloires", promulgada en marzo de 1991 y que ya ha sido suscrita por más de cuatrocientas universidades alrededor del mundo (Anexo B). En esta declaración, las universidades firmantes se comprometen con un plan de acción específico para apoyar la creación de esa nueva forma de vida, no solo en su plataforma académica sino, siendo ellas mismas, en su gestión, modelos de desarrollo sustentable en el uso de los recursos, consumo de energía, manejo de desechos y otros aspectos propios de su actividad cotidiana.

Los firmantes de esta declaración se han comprometido a llamar la atención de los distintos actores de la sociedad sobre la necesidad de crear un futuro ambientalmente sostenible, incentivar al interior de sus instituciones la investigación, la docencia y la extensión en temas relacionados con el medio ambiente y el desarrollo, así como también a ser modelos, ellas mismas, de responsabilidad ambiental, estableciendo los programas de conservación de recursos, reciclaje y reducción de desechos dentro de la universidad.

Las instituciones académicas de alto nivel tienen la capacidad de orientar e influir en 
el destino de la sociedad de la que forman parte. El currículo es formado por los elementos que las personas expertas en cada disciplina consideran son el campo de acción del presente y futuro de una profesión y principalmente, por las valoraciones subjetivas que dichas personas poseen; al traducirse estas valoraciones en el conjunto de conocimientos habilidades y actitudes que componen el plan de estudios se conforma, mediante el proceso de enseñanza aprendizaje, a los futuros líderes de la sociedad.

\section{RECOMENDACIONES PARA LA ADECUACIÓN DE LOS CURRÍCULOS UNIVERSITARIOS A LA EDUCACIÓN PARA LA SOSTENIBILIDAD}

Con base en el análisis anteriormente expuesto, se pueden desprender de él algunas recomendaciones para la revisión del currículo universitario que contribuyan a una educación para la educación sostenible de los futuros líderes de la sociedad:

- Promover la interdisciplinariedad, integrando la investigación y la docencia y la acción social con la toma de decisiones; apoyar el intercambio académico y la vinculación con la práctica en situaciones reales donde el aporte debe caracterizarse por su integralidad, derivada de la interdisciplina.

- Promover y apoyar la investigación orientada a la sostenibilidad en todos los campos del conocimiento.

- Integrar, en cada facultad, equipos interdisciplinarios que desarrollen programas que promuevan la interdisciplina en docencia, investigación y acción social.

- Apoyar los esfuerzos de los estudiantes en proyectos orientados hacia la sostenibilidad, mediante la creación de un centro de apoyo a las iniciativas que presenten.
- Generar el debate público sobre esta problemática.

- Modificar las prácticas de gestión de las universidades para que sean modelos de sostenibilidad ante la sociedad y rendir informes públicos sobre los logros alcanzados.

- Establecer un plan de acción con objetivos por alcanzar en el corto, mediano y largo plazo, dirigido a cumplir las metas de la Década para la Educación para la Sostenibilidad.

Las causas de la crisis que afectan la vida en el planeta, entre las que se encuentran, la sobreexplotación de los recursos, la contaminación, el modelo económico basado en el consumo, la pobreza, la desigualdad, la discriminación y la enfermedad y muerte causadas por causas prevenibles, son parte de nuestro diario vivir, el reto ahora es no acostumbrarnos a ellas; las universidades y quienes en ellas laboramos, tenemos especial responsabilidad en contribuir con la superación de la crisis creando nuevas condiciones para un desarrollo sustentable y equitativo para todos.

\section{RECOMENDACIONES ESPECÍFICAS PARA LOS CURRÍCULOS DE LAS CARRERAS DE INGENIERÍA}

La ingeniería ha desarrollado la infraestructura básica de la que depende la sociedad actual; el transporte, los servicios básicos, la vivienda, la producción de nuevos materiales, la producción y distribución de alimentos, las obras de infraestructura, la generación de energía, entre otros, son algunos de los ejemplos más claros de cómo la intervención de la ingeniería ha modelado nuestro estilo de vida.

Sin embargo, junto con estos desarrollos, también se han producido algunos efectos indeseables, que afectan , como ya se ha visto en este trabajo, el medio ambiente y la salud de las personas e incluso, están llegando a niveles donde algunos 
expertos opinan que el daño ya no es reversible, este fenómeno se ha acelerado principalmente en los últimos cincuenta años.

Las ciudades con sus aparentes oportunidades en servicios básicos, acceso a centros de salud, educación, cultura y oportunidades de progreso social y económico han servido de imanes para la población rural y se calcula que para este año, por primera vez en la historia, más gente vivirá en las ciudades que en el campo, poniendo una presión extraordinaria en la capacidad de ofrecer servicios básicos, que provean alimentación, agua, vivienda, empleo y satisfagan las demás necesidades de una población creciente (UNPF, 2005).

En los próximos veinte o treinta años, se deben crear dos billones de empleos, uno de ellos para los desempleados y subempleados actuales y otro para los jóvenes que se incorporarán al mercado laboral en este período, este objetivo no puede ser alcanzado con el modelo económico vigente que sustituye el trabajo por capital, consume grandes cantidades de materiales y de energía y genera también volúmenes crecientes de basura y desperdicios, de acuerdo con (Cortese, 1998): "Nuestro pensamiento está equivocado: nosotros usamos más de lo que tenemos menos (recursos naturales), y usamos menos de lo que tenemos más (fuerza de trabajo)".

En cuanto a los currículos para las carreras de ingeniería, conviene hacer algunas recomendaciones particulares:

- La formación de los futuros ingenieros debe superar el esquema actual que los capacita para trabajar con un aspecto específico de la problemática ambiental; problemas como contaminación, polución del aire o del agua, desechos tóxicos, son parte del currículo actual, pero no se contempla el trabajo con una problemática ambiental compleja desde un enfoque integrado y comprensivo, el énfasis actual está puesto en el estudio de los métodos para controlar los desechos o la polución, más que en las opciones para reducir o eliminar la generación de polución o desechos desde el punto en el que se producen.
- En un nuevo enfoque se debe trabajar sobre la base de cambiar los procesos y sistemas de producción y consumo, más que en administrar los problemas que genera un modo de vida insostenible.

- $\quad$ La ingeniería debe jugar un papel mucho más proactivo en la definición de las políticas públicas que provean al sector productivo de un marco sostenible de desarrollo, diseñado para funcionar en el largo plazo; de no hacerlo, este liderazgo puede ser ocupado por otros profesionales que no poseen la formación y los conocimientos necesarios para comprender los límites de la tecnología y sus consecuencias en el marco de la sostenibilidad.

- El currículo de las escuelas y facultades de ingeniería debe prestar especial atención al problema de la generación y consumo de energía, diseñando tecnologías más eficientes y abriendo oportunidades a la sustitución de los combustibles fósiles por fuentes alternativas de energía, así como también incorporando la investigación y estudio de mecanismos para reducir los patrones de consumo actuales.

- Se debe enfatizar el trabajo interdisciplinario con docentes y estudiantes de otros campos del conocimiento, para que la formación que se ofrezca a los estudiantes incluya una visión integral de los problemas y no fragmentos como ocurre actualmente, de esta manera, aprenderán a elaborar diseños y propuestas que consideren la integralidad de la solución.

- El nuevo currículo debe estar centrado en la formulación de la pregunta, más que en la búsqueda de respuestas a preguntas que no se sabe quién planteó; se debe enfatizar en la formación de valores más que en teorías estudiadas sin contexto, enfatizar más en la toma de conciencia que en la sola abstracción del dato que nada dice de la realidad, preocuparse y trabajar más en la formación de un compromiso con la sociedad y no 
solamente en la eficiencia de una solución técnica, sin que ello signifique desprecio por una solución técnica, correctamente aplicada.

- El nuevo currículo debe ser una nueva aproximación; no podemos arreglar un modelo que ya alcanzó sus límites, es necesario crear un nuevo modelo que, basado en una educación para la sostenibilidad, fortalezca el respeto por el medio ambiente, procure la eliminación de las desigualdades y ponga a la persona como centro de la creación y la única responsable de su destino.

Como enseña la filosofía china, una crisis está compuesta por dos elementos, un peligro y una oportunidad, la crisis que tenemos ante nosotros es indudablemente un gran riesgo para el futuro de la vida en el planeta; en nosotros está convertirla en una oportunidad en beneficio quienes en él habitamos y de quienes habrán de venir después de nosotros.

\section{危机}

Fuente: (Crisis en idioma mandarín. Traductor de internet)

\section{REFERENCIAS BIBLIOGRÁFICAS}

Capra, F. (1985). El punto crucial, el integral. Barcelona. Editorial Integral.

Capra, F. (2003). Las conexiones ocultas: implicaciones sociales, medioambientales, económicas y biológicas de una nueva visión del mundo. Barcelona. Editorial Anagrama.
Chacón, R. Uso de hidrocarburos en transporte y contaminación en Costa Rica: Instituto Meteorológico Nacional. Extraído el 24 de enero del 2005 de www.imn.ac.cr.

Chin-Wo, A. (2005). Energía y Materia: Oportunidades y barreras para el desarrollo de las energía renovables en Costa Rica. Conferencia presentada en el CIENTEC. Extraído el 24 de enero del 2005 de www. cientec.or.cr.

CENEAM: (Centro Nacional de Educación y Formación Ambiental. Ministerio del Medio Ambiente). Década de las Naciones Unidas de la Educación para el Desarrollo Sostenible. Extraído el 23 de junio del 2005 de http://www.mma.es/educ/ceneam/ 10documentos/otros/decada.htm.

Cortese, A. (1999). Education for sustainability: the need for a new human perspective. Second Nature. Extraído el 9 de febrero del 2005 de www.secondnature.org.

Cortese A. (1998). The role of engineers in creating an environmental sustainable future. Second Nature. Extraído el 9 febrero del 2005 de www.secondnature. org.

Cortese, A. (1970). Unprecedented challenges to human prosperity and survival in the twentyfirst century. Extraído el 16 de agosto del 2005 de http://www.secondnature.org. inf@secondnature.org.

AssociationofUniversityLeadersforaSustainable Future. Declaración de Talloires. Extraído el 16 de agosto del 2005 de www.ulsf.org/ pdf/Spanish_TD.pdf .

Ehrenfeld, D. (2001). A Techno-pox upon the land. Harper's Magazine. En Estado de la Nación: Proyecto Estado de la Nación en Desarrollo Humano Sostenible. San José, Costa Rica. 
Einstein, A. Citas y refranes. Extraído el 19 de abril del 2005 de www.citasyrefranes. com.

Halweil, B. (2002). Home Grown: The case for local food in a global market. Wordwatch paper 163.

INBIO (Instituto Nacional de Biodiversidad). Extraído el 16 de agosto del 2005 de www. inbio.ac.cr.

IPCC, WG1 Intergovernmental Panel on Climate Change, Working Group 1. Third Assessment Report, Summary for policymakers. Extraído el 19 de abril del 2005 de www.ipcc.ch.

OEI (Organización de Estados Iberoamericanos). Década por una educación para la sostenibilidad. Extraído el 14 de marzo del 2005 de www.oei.es/decada/.

ONU (Organización de las Naciones Unidas). Resolución 57/254: Década de las Naciones Unidas de la Educación para el Desarrollo Sostenible. Extraído el 7 febrero del 2005 de www.onu.org.

Ryan, J. (1997). Stuff: The secret lives of everyday things, Northwestern Environment Watch.

UNDP (United Nation Development Programmer).(2001).HumanDevelopment Report, IPCC WGI Third Assessment Report, Shanghai Draft, Summary for Policymakers. Extraído el 19 de abril del 2005 de www.undp.org/hdr2003/.

UNPD The United Nations Environment Programmed (UNEP), the World Bank, the World Resources Institute: A Guide to World Resources 2000-2001: People and Ecosystems: The Fraying Web of Life, 2000

UNICEF (United Nations Children's Fund). Extraído el 28 de febrero del 2005 de www.unicef.org.

UNPF (United Nations Population Fund). Extraído el 28 febrero del 2005 de www. unfpa.org.

USEPA(United States Environmental Protection Agency). (2000). Global Warming and Our Changing Climate, April. Extraído el 28 de febrero del 2005 de www.epa.you/ safewater/.

Buscador Google. Extraído el 25 de agosto del 2005 de http://translate.google.com/ translate_t

Observatorio de la NASA. Extraído el 25 de agosto del 2005 de http://earthobservatory. nasa.gov/Newsroom/New Images / images.php3?img_id=1011.

\section{SOBRE LA AUTORA}

\section{Herminia Casanueva López}

Licenciada en Psicología de la UNAM, México M. Sc. en Ciencias de la Educación Profesora de la Escuela de Ingeniería Agrícola Facultad de Ingeniería, Universidad de Costa Rica.

Teléfono: (506) 2075682

Facsímil: (506) 2074614

Correo electrónico: hcasanueva@osa.ingagri. ucr.ac.cr 


\begin{abstract}
ANEXO A
Década de las Naciones Unidas de la Educación para el Desarrollo Sostenible

Resolución 57 / 254 aprobada por la Asamblea General de las Naciones Unidas,
\end{abstract}

20 de diciembre de 2002

\title{
La Asamblea General
}

Recordando el capítulo 36 del Programa 21 relativo al fomento de la educación, la capacitación y la toma de conciencia, aprobado en la Conferencia de Naciones Unidas sobre el Medio Ambiente y el Desarrollo, celebrada en Río de Janeiro, Brasil en 1992

Reafirmando el objetivo de desarrollo acordado a nivel internacional de lograr la educación primaria universal, en particular que, para el año 2015, los niños y las niñas de todo el mundo puedan terminar un ciclo completo de enseñanza primaria.

Expresando su reconocimiento por la contribución hecha por la Comisión sobre el Desarrollo Sostenible al tratamiento de la cuestión de la educación para el desarrollo sostenible desde la celebración de la Conferencia de las Naciones Unidas sobre el Medio Ambiente y el Desarrollo.

Acogiendo complacida el hecho de que el Plan de Acción de las Decisiones de la Cumbre Mundial sobre el Desarrollo Sostenible, conocido como "Plan de acción de Johannesburgo", se haya confirmado la importancia de la educación para el desarrollo sostenible y recomendado que la Asamblea General considerase la posibilidad de proclamar un decenio de la educación para el desarrollo sostenible a partir de 2005.

Subrayando que la educación es un elemento indispensable para alcanzar el desarrollo sostenible.

1. Decide proclamar el período de diez años que comienza el $1^{\circ}$ de enero de 2005 Década de las Naciones Unidas de la Educación para el Desarrollo Sostenible.

2. Designa a la Organización de las Naciones Unidas para la Educación, la Ciencia y la Cultura (UNESCO) organismo rector de la promoción de la Década y le pide que prepare un proyecto de plan de acción internacional, en el que se aclare la relación de la Década con los proyectos educacionales en curso, en particular el Marco de Acción de Dakar, aprobado en el Foro Mundial sobre Educación y el Decenio de las Naciones Unidas sobre Alfabetización, en consulta en Naciones Unidas y otras organizaciones internacionales competentes, gobiernos, organizaciones no gubernamentales y otras entidades interesadas, con miras a presentar recomendaciones a los gobiernos sobre la forma de promover y mejorar la integración de la educación para el desarrollo sostenible en sus respectivos planes de acción y estrategias en materia de educación al nivel apropiado.

3. Invita a los gobiernos a que consideren incluir medidas para aplicar la Década en sus respectivos planes de acción y estrategias en materia de educación a más tardar para el año 2005, teniendo en cuenta el plan de acción internacional que ha de preparar UNESCO.

4. Decide incluir en el programa provisional de su quincuagésimo octavo periodo de sesiones un tema titulado "Década de las Naciones Unidas de la Educación para el Desarrollo Sostenible" 


\section{ANEXO B}

\section{Declaración de Líderes de Universidades para un Futuro Sostenible}

Association of University Leaders for a Sustainable Future

\section{DECLARACIÓN DE TALLOIRES}

Nosotros, los rectores, vicerrectores, y vicecancilleres de las universidades de todo el mundo, estamos conscientes del rápido e impredecible crecimiento de la contaminación, de la degradación del medio ambiente y del agotamiento de los recursos naturales. De hecho, la contaminación del agua y del aire local, regional y global, la destrucción y la disminución de bosques, suelos y agua, la reducción de la capa de ozono y la emisión de gases contaminantes peligran en contra la supervivencia de los seres humanos y especies vivientes, la integridad de la tierra y su biodiversidad, la seguridad de las naciones y en contra de la herencia que permanecerá para las futuras generaciones.

Estos cambios en el medio ambiente son causados por una producción desequilibrada e insostenible y por patrones de consumo que agravan los niveles de pobreza en muchas regiones del mundo.

Creemos así, que se requieren urgentes acciones para combatir y revertir la tendencia de los problemas anteriormente mencionados.

De esta forma, la estabilización de la población humana, la adopción de tecnologías agrícolas e industriales ambientalmente sanas, la reforestación y la restauración ecológica, son elementos esenciales en la creación de un futuro equilibrado y sostenible para que la humanidad esté en armonía con la naturaleza. Las universidades tienen un papel importante en la educación, investigación, formación de políticas y en el intercambio de información necesaria para alcanzar estos objetivos.

Las universidades deben proporcionar así, el liderazgo y el apoyo para movilizar los recursos internos y externos, de modo que sus instituciones respondan a este urgente desafío. Nosotros, por lo tanto, hemos acordado en ejercer las siguientes acciones:

1. Aprovechar cada oportunidad para despertar la conciencia del gobierno, las industrias, las fundaciones y las universidades en expresar públicamente la necesidad de encaminarnos hacia un futuro ambientalmente sostenible.

2. Incentivar a la universidad para que se comprometa con la educación, investigación, formación de políticas e intercambios de información de temas relacionados con población, medio ambiente y desarrollo y así alcanzar un futuro sostenible.

3. Establecer programas que formen expertos en gestión ambiental, desarrollo sostenible, demografía y temas afines para asegurar así que los egresados universitarios tengan una capacitación ambiental y sean ciudadanos responsables.

4. Crear programas que desarrollen la capacidad de la universidad en enseñar el tema del medio ambiente a estudiantes de pregrado, postgrado e institutos profesionales.

5. Ser un ejemplo de responsabilidad ambiental estableciendo programas de conservación de los recursos, reciclaje y reducción de desechos dentro de la universidad. 
6. Involucrar al gobierno (en todos los niveles), a las fundaciones y a las industrias, en el apoyo a la investigación universitaria, educación, formación de políticas e intercambios de información sobre desarrollo sostenible. Extender también este trabajo a las organizaciones no gubernamentales (ONG) y encontrar así soluciones más integrales a los problemas del medio ambiente.

7. Reunir a los profesionales del medio ambiente para desarrollar programas de investigación, formación de políticas e intercambios de información para alcanzar de esta forma un futuro ambientalmente sostenible.

8. Asociarse con colegios de educación básica y media para capacitar a sus profesores en la enseñanza de problemas relacionados con población, medio ambiente y desarrollo sostenible.

9. Trabajar con la Conferencia de las Naciones Unidas para el Medio Ambiente y el Desarrollo, CNUMAD, El Programa de las Naciones Unidas para el Medio Ambiente, PNUMA y otras organizaciones nacionales e internacionales para promover un esfuerzo universitario a nivel mundial que conlleve a un futuro sostenible.

10. Establecer un Comité Directivo y un Secretariado para continuar esta iniciativa y para informarse y apoyarse los unos a los otros en el cumplimiento de esta Declaración. 